\title{
Availability Maps for Connections in WDM Optical Networks
}

\author{
Darli A. A. Mello*, Dominic A. Schupke ${ }^{\dagger}$, Matthias Scheffel ${ }^{\ddagger}$ and Helio Waldman* \\ * Optical Networking Laboratory - State University of Campinas - UNICAMP \\ Av. Albert Einstein 400, CP 6101, CEP 13083-970 - Campinas - SP - Brazil \\ Email: \{darli,waldman\}@ decom.fee.unicamp.br \\ ${ }^{\dagger}$ Siemens Corporate Technology \\ Otto-Hahn-Ring 6, 81730 Munich, Germany \\ Email: dominic.schupke@siemens.com \\ $\ddagger$ Munich University of Technology, Institute of Communication Networks \\ Arcisstr. 21, 80333 Munich, Germany \\ Email: matthias.scheffel@tum.de
}

\begin{abstract}
The provisioning of services in optical networks demands reliable transmission paths. This paper investigates the availability of optical connections with respect to the failure probability of the involved network components. Outages of the fiber links as well as potential hardware failures for different opaque and transparent node architectures are analyzed. The results are presented in terms of map diagrams which allow to determine the availability of a wavelength connection subject to the transmission distance and the number of traversed hops.

Index Terms-Availability Maps, Availability, Reliability.
\end{abstract}

\section{INTRODUCTION}

The availability of services is an important issue in today's optical networks. Because of the enormous bandwidth of the fiber medium, data can be transmitted simultaneously on a great number of different frequencies at high bitrates. This wavelength division multiplexing (WDM) technology thus allows ultra high transmission rates per fiber. In case of a fiber or node component failure, individual or multiple lightpaths within one fiber can be interrupted, which affects several data connections. To be prepared for such incidents, precautions are taken in order to rapidly restore the optical transmission. The task of providing resilience is addressed in a number of papers. Often the focus lies on the consideration of link failures solely, which are caused by fiber cuts during digging work for example. The provisioning of an alternative transmission path in addition to the primary one using no common fiber edge can compensate single link failures. However, the outage of optical fiber is not the only conceivable failure scenario. Each hardware component involved in the transmission path goes down with a certain probability. Network providers promise to their business customers an availability of network access in terms of service level agreements (SLAs) of the contract [1]. If the connection resilience requirement cannot be achieved with an unprotected single path, protection or restoration mechanisms may be necessary. In any case, in order to guarantee the desired level of resilience the entire data transmission path must be analyzed. The wavelength routing and signal refreshing processes involve a great number of hard- ware components such as transceivers, multiplexers, switches, amplifiers, and regenerators, which inevitably contribute to the overall reliability.

This paper investigates the availability of connections routed in an optical network considering the joint impact of fiber medium failures as well as network component failures. Previous publications that approached this field regarded nodes as atomic entities characterized by a single availability value [2] [3]. References [4], [5], and [6] emphasize the availability performance of specific node architectures. Reference [7] performs a sensitivity analysis of component failures in the circuit switched WDM network. This work analyzes different node architectures and identifies the connection availability impairments related to adding, dropping, or just letting the path pass through the node. Opaque and transparent structures are compared. As for the transparent architectures, the availability degradation due to a potential lightpath regeneration in a pool of OEO regenerators, which has been viewed as an alternative to overcome the physical impairments inherent to the transparent transmission [8], has been considered. We visualize the probability for an intact connection transmission in terms of map diagrams subject to the connection length and the number of traversed nodes. These availability maps are universally applicable to any network topology and allow to easily verify the availability performance of a connection of any distance and hop count.

The rest of this paper is organized as follows. Section II explains how the component failure rates adopted along the paper have been estimated. Section III derives availability block diagrams for several node architectures assembled with the components described in Section II. Section IV presents and analyzes availability maps of connections whose lightpaths traverse the node architectures presented in the previous section. Finally, Section V concludes the paper.

\section{Component Failure Rates}

Component failure rates are often sensitive data which are difficult to find in the literature. The following component fail- 
ure rates rely on previous publications and educated guesses. Indeed, this study does not aim at providing exact values for component availability, but to offer consistent estimates which allow to verify the joint impact of node architectures and fiber links on the connection availability. This paper assumes that the arrival and departure (repair) of component failures are memoryless processes with constant means, and that the failure rate in FIT ( 1 FIT $=$ a failure rate of 1 failure in $\left.10^{9} h\right)$ of a series system is the sum of the failure rates in FIT of the constituting components.
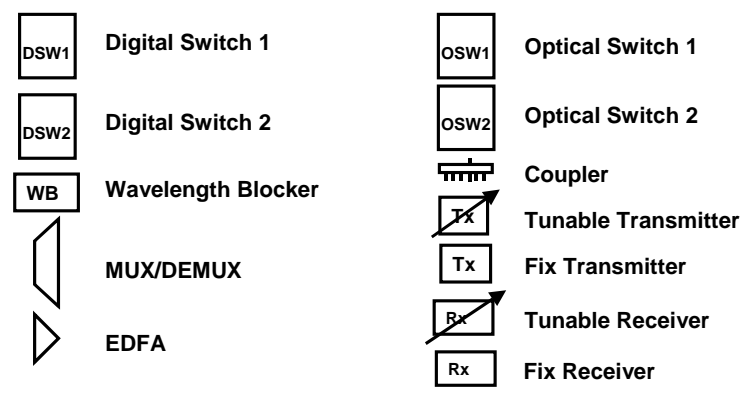

Figure 1: Component symbols.

The symbol of each component can be found in Figure 1, and their failure rates are summarized in Table I. The failure rate of node components were estimated according to the following assumptions:

\section{MUX/DEMUX}

The failure rate was assumed to be proportional to the number of supported wavelengths. The basic value was obtained from [5] (100 FIT for 4 wavelengths and 200 FIT for 8 wavelengths).

\section{EDFA}

The failure rate of node-internal erbium doped fiber amplifiers (EDFAs) was obtained from [4]. The failure rate of inline EDFAs is included in the link failure rate.

\section{Optical Switch 1}

The Optical Switch 1 is an optical switch matrix with add/drop capability based on two-dimensional microelectromechanical systems (2D-MEMS). Reference [9] demonstrated an optical add/drop multiplexer (OADM) which uses such switch matrix with WxM switch mirrors, where $\mathrm{W}$ is the number of incoming lightpaths to be switched, and $\mathrm{M}$ is the number of add/drop ports. In this paper we assume $\mathrm{M}=\mathrm{W} / 4$ since only part of the traffic may be dropped. Reference [10] verifies an estimate of less than 21 FIT for the random failure rate of a switch element under static reliability tests, which lets us assume a failure rate of $21 * \mathrm{~W} * \mathrm{~W} / 4$ FIT.

\section{Optical Switch 2}

The Optical Switch 2 is an optical 3D-MEMS based switch device suitable for optical crossconnects (OXCs) with wavelength selective architecture [11]. The majority of commercial 3D-MEMS switch designs use a number of mirrors which is twice the number of input ports [12]. In the wavelength selective architecture each optical switch device has $2 \mathrm{~N}$ input and output ports to allow full add/drop capability, where $\mathrm{N}$ is the number of incoming fibers. Using again reference [10] a failure rate of $21 * 2 * 2 \mathrm{~N}$ FIT was assumed.

\section{Coupler 1}

Coupler 1 is a 1:2 power splitter. The failure rate of couplers was assumed to be proportional to the number of outputs. The basic value was obtained from [6], which considers a failure rate of 50 FIT for a 1:2 splitter.

\section{Coupler 2}

Coupler 2 is a $1: \mathrm{W} / 4$ power splitter, where $\mathrm{W}$ is the number of wavelengths supported by each incoming fiber.

\section{Coupler 3}

Coupler 3 is a $1:(\mathrm{N}-1)$ power splitter, where $\mathrm{N}$ is the number of incoming fibers to the OXC.

\section{Tunable Transmitter}

The failure rate was obtained from [6].

Fix Transmitter

The failure rate was obtained from [6].

\section{Tunable Receiver}

The tunable receiver is able to select a specific channel, and to filter others. The failure rate was obtained from [6].

\section{Fix Receiver}

The failure rate was obtained from [6].

\section{Digital Switch 1}

The Digital Switch 1 is a digital switch matrix with add/drop capability, suitable for opaque OADMs supporting $\mathrm{W}$ wavelengths. This paper assumes the failure rate as proportional to the number of input channels. The basic value was obtained from [4] (3500 FIT for a $4 \times 4$ switch).

\section{Digital Switch 2}

The Digital Switch 2 is a digital switch matrix with add, drop, and crossconnect capability, suitable for opaque OXCs supporting $\mathrm{WxN}$ channels, where $\mathrm{W}$ is the number of wavelengths carried by each of the $\mathrm{N}$ incoming fibers. Again we assume a failure rate that is proportional to the number of input channels. The basic value was obtained from [4] (3500 FIT for a $4 \times 4$ switch).

\section{Wavelength Blocker}

This paper assumes that the failure rate is proportional to the number of supported wavelengths such as for a multiplexer and a demultiplexer, which are important components of wavelength blockers. Some wavelength blockers may also be used as channel equalizers.

\section{Node Architectures}

Transparent optical nodes enable the routing of hundreds of channels, in turn carrying in the order of gigabits per second, through a communications network. Some advantages over opaque alternatives include transparency to the bitrate, scalability and ability to transport any higher layer protocol. Moreover, typically a large portion of traffic passes through a node, thus making optical transparency attractive. For instance, approximately $75 \%$ of the traffic passes trough a node of 
Table I: Component Failure Rates. W: Number of wavelengths per fiber. N: Number of incoming fibers.

\begin{tabular}{|l|l|l|}
\hline Component & Symbol & Failure Rate [FIT] \\
\hline MUX/DEMUX & MUX & $25 * \mathrm{~W}$ \\
EDFA & EDFA & 2850 \\
Optical Switch 1 & OSW1 & $21 * \mathrm{~W} * \mathrm{~W} / 4$ \\
Optical Switch 2 & OSW2 & $21 * 2 * 2 \mathrm{~N}$ \\
Coupler 1 & COUP1 & $25 * 2$ \\
Coupler 2 & COUP2 & $25 * \mathrm{~W} / 4$ \\
Coupler 3 & COUP3 & $25 *(\mathrm{~N}-1)$ \\
Tunable Transmitter & TTx & 745 \\
Fix Transmitter & FTx & 186 \\
Tunable Receiver & TRx & 470 \\
Fix Receiver & FRx & 70 \\
Digital Switch 1 & DSW1 & $875 * \mathrm{~W}$ \\
Digital Switch 2 & DSW2 & $875 * \mathrm{~W} * \mathrm{~N}$ \\
Wavelength Blocker & WB & $50 * \mathrm{~W}$ \\
\hline
\end{tabular}

typical nationwide network systems in the North American region [13], and a similar phenomenon is observed with the Internet traffic in European networks [3]. This evidences the benefits of all-optical switching in which the costly electronic processing is avoided. Therefore this paper investigates the performance in terms of availability of two important transparent architectures, and in addition one opaque case.

\section{A. Wavelength Selective OADM}

The wavelength selective (WS) architecture for OADMs is based on a demultiplexer, followed by a switch fabric interconnecting express and add/drop ports, and a multiplexer [11] [14]. These architectures do not consider the utilization of channel equalizers, though they may be necessary. Reference [9] demonstrated a micro-machined OADM with client reconfigurability, which is the capability for the client to add/drop any wavelength to any add/drop port. Figure 2 shows the basic structure of a MEMS based WS OADM and the trajectories followed by a connection inside the node. If the accumulated impairments on an optical channel become excessive, there is a possibility to electrically regenerate it by dropping and consecutively adding the connection. The block diagrams used for calculating the availability penalty of each node operation are found in Figure 3. Note that the transmitters have to be tunable to allow client reconfigurability.

\section{B. Broadcast and Select OADMs}

The broadcast and select architecture (B\&S) for OADMs is based on passive splitters/couplers, tunable filters and optical amplifiers to compensate for losses [11] [14]. Reference [13] reports experiments with $\mathrm{B} \& S$ OADMs which exhibit low loss for pass through channels, achieving greater distances without regeneration. Figure 4 shows the basic structure of a B\&S OADM and the trajectories followed by a connection inside the node. Again, if the accumulated impairments on an optical channel become excessive, there is a possibility to electrically regenerate it by dropping and consecutively adding the connection. The block diagrams used for calculating the availability penalty of each node operation can be found in Figure 5.

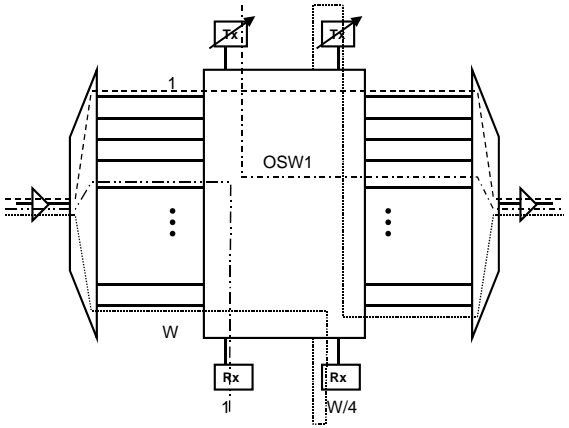

Figure 2: WS OADM. Availability penalties due to the operations of regeneration (dotted line), passing through (dashed line), adding (dashed single-dotted line) and dropping (dashed double-dotted line).

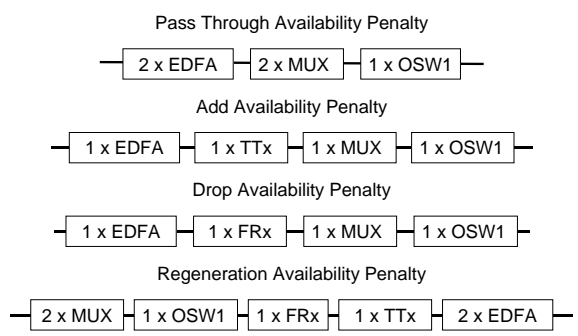

Figure 3: WS OADM. Block diagrams for the calculation of availability penalties.

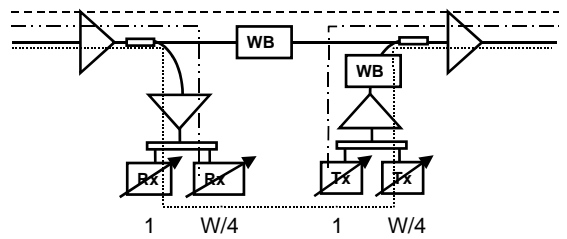

Figure 4: B\&S OADM. Availability penalties due to the operations of regeneration (dotted line), passing through (dashed line), adding (dashed single-dotted line) and dropping (dashed double-dotted line).

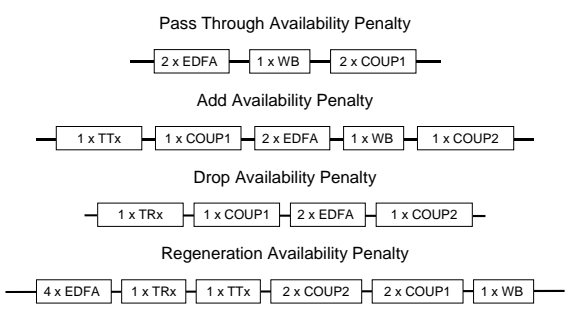

Figure 5: B\&S OADM. Block diagrams for the calculation of availability penalties.

\section{Opaque OADMs}

Figure 6 shows the basic structure of an opaque OADM, and the trajectories followed by a connection inside the 
node. Regeneration and wavelength conversion are intrinsic to opaque OADMs. The block diagrams used for calculating the availability penalty of each node operation can be found in Figure 7.

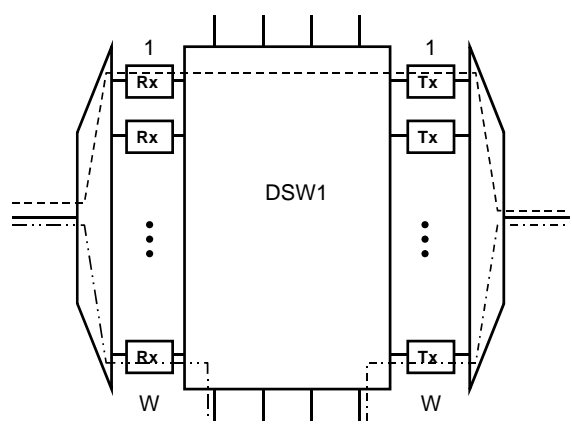

Figure 6: Opaque OADM. Availability penalties due to the operations of passing through (dashed line), adding (dashed single-dotted line) and dropping (dashed double-dotted line).

Pass Through Availability Penalty
Add Availability Penalty
Drop Availability Penalty
$1 \times \mathrm{FRx}-1 \times \mathrm{FTx}-2 \times \mathrm{FUX}-1 \times \mathrm{FSW} 1$ - $1 \times 1 \times \mathrm{MUX}-1 \times \mathrm{DSW} 1$

Figure 7: Opaque OADM. Block diagrams for the calculation of availability penalties.

\section{Wavelength Selective OXCs}

The wavelength selective (WS) architecture for OXCs makes use of the wavelength continuity constraint to reduce the complexity of its switch mechanism. Since two channels using the same wavelength but coming to the OXC from different fibers cannot be simultaneously switched into the same fiber, the switch mechanism does not have to provide full switching capability from all incoming channels to all outgoing channels. The WS architecture dedicates a separate optical switch device to each wavelength [14][11], as depicted in Figure 8. These architectures do not consider the utilization of channel equalizers, though they may be necessary. The switch device has $2 \mathrm{~N}$ input ports and $2 \mathrm{~N}$ output ports, where $\mathrm{N}$ is the number of incoming fibers, to allow any channel to be added or dropped. One advantage of the wavelength selective architecture over using a single switch matrix, in terms of connection reliability, is the ability to repair each switch device separately. This paper assumes a 3D-MEMS based switch device for the WS OXC architecture. The block diagrams used for calculating the availability of each node operation can be found in Figure 9 .

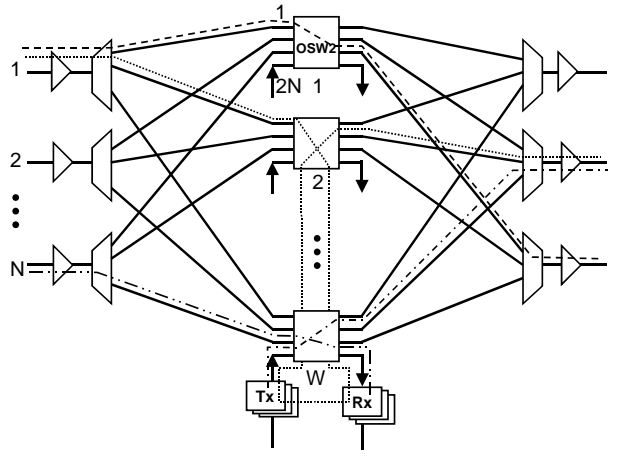

Figure 8: WS OXC. Availability penalties due to the operations of regeneration (dotted line), passing through (dashed line), adding (dashed single-dotted line) and dropping (dashed double-dotted line).

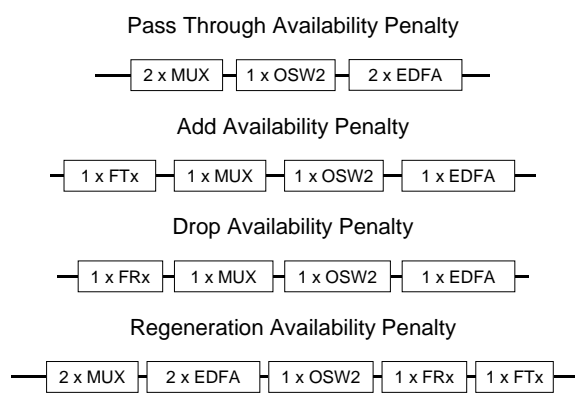

Figure 9: WS OXC. Block diagrams for the calculation of availability penalties.

\section{E. Broadcast and Select OXCs}

The broadcast and select architecture (B\&S) for OXCs is based on passive splitters/couplers, tunable filters and amplifiers to compensate for losses [11] [14]. Reference [13] reports experiments with B\&S OXCs. Figure 10 shows the basic structure of a B\&S OXC and the trajectories followed by a connection inside the node. If the accumulated impairments on an optical channel become excessive, there is a possibility to electrically regenerate it by dropping and adding the connection. The block diagrams used for calculating the availability of each node operation can be found in Figure 11.

\section{F. Opaque OXCs}

Figure 12 shows the basic structure of an opaque OXC, and the trajectories followed by a connection inside the node. Opaque OXCs based on opto-electrical interfaces and electrical switching technology offer intrinsic benefits such as $3 \mathrm{R}$ regeneration and wavelength conversion. The block diagrams used for calculating the availability of each operation performed by the node can be found in Figure 13.

\section{AVailability Maps}

Availability maps are connection availability contour plots that are function of the connection length and of the number 


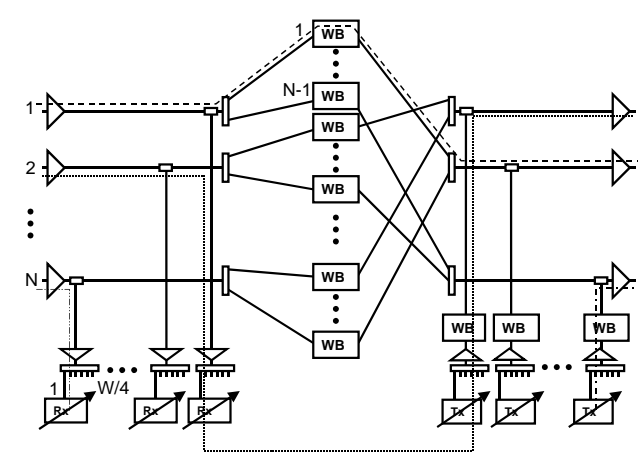

Figure 10: B\&S OXC. Availability penalties due to the operations of regeneration (dotted line), passing through (dashed line), adding (dashed single-dotted line) and dropping (dashed double-dotted line).

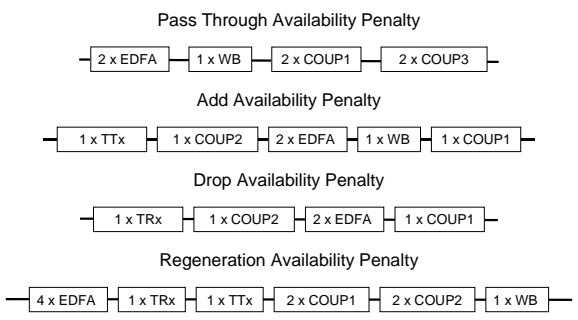

Figure 11: B\&S OXC. Block diagrams for the calculation of availability penalties.

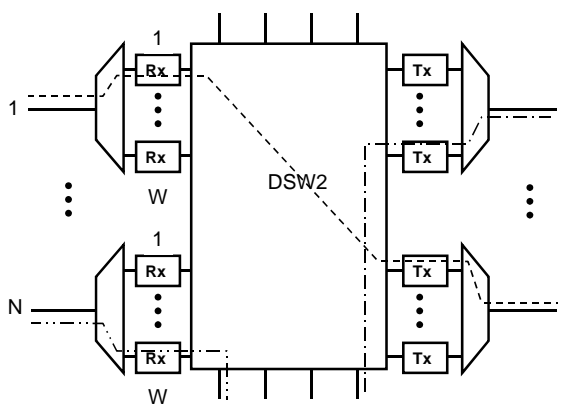

Figure 12: Opaque OXC. Availability penalties due to the operations of passing through (dashed line), adding (dashed single-dotted line) and dropping (dashed double-dotted line).

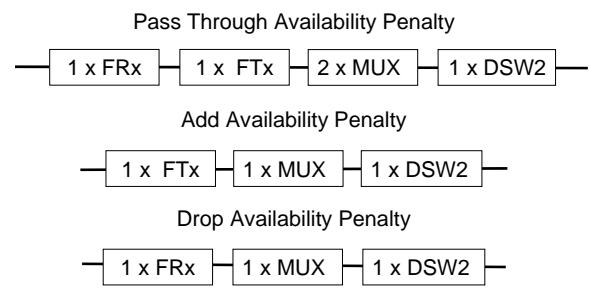

Figure 13: Opaque OXC. Block diagrams for the calculation of availability penalties. of traversed hops. They provide generic results that allow to quantify and visualize the joint influence of node and link failures on the connection availability in any network. Some typical values for the connection length and the number of traversed hops are: a maximum of 9 hops and $5819 \mathrm{~km}$ for a hypothetical European optical network [15]; an average connection length of 3.48 hops for a sparse hypothetical panEuropean optical network [3]. Typical values for a North American network can be found in [16]. The connection availability $A_{c}$ is estimated by Equation 1:

$$
\begin{aligned}
A_{c}=1 & -\left(A P_{\text {link-km }} D+\right. \\
& +A P_{\text {drop }}+ \\
& +A P_{\text {add }}+ \\
& +A P_{\text {pass-thr }}\left\lceil(N H-1)\left(1-P_{\text {reg }}\right)\right\rceil+ \\
& \left.+A P_{\text {reg }}\left\lfloor(N H-1) P_{\text {reg }}\right\rfloor\right),
\end{aligned}
$$

where $A P_{l i n k-k m}$ is the link availability penalty per kilometer, $D$ is the connection length in kilometers, $N H$ is the number of hops traversed by the connection, $A P_{a d d}$, $A P_{\text {drop }}, A P_{\text {pass-thr }}, A P_{\text {reg }}$ are the availability penalties due to the adding, dropping, passing through and regeneration node operations, respectively. $P_{r e g}$ is the fraction of nodes in which the connection is regenerated. Here $\lceil x\rceil$ rounds a real number $x$ to the nearest integer towards infinity, and $\lfloor x\rfloor$ rounds $x$ to the nearest integer towards minus infinity.

In general, the availability penalty imposed by a system can be derived from its failure rate $F R$ in FIT by Equation 2:

$$
A P=10^{-9} * F R * M T T R,
$$

where MTTR is the mean time to repair in hours.

This paper assumes a link failure rate per kilometer of $310 \mathrm{FIT} / \mathrm{km}$ [17] that includes fiber and inline amplifier failures. Here we do not consider the possible deployment of Raman-pumped amplifiers, which involve less amplifiers, thus making the link less failure-prone. The link MTTR was assumed to be $12 \mathrm{~h}$. The node availability penalties were estimated using the block diagrams and failure rates presented in the previous sections. The component MTTR was assumed to be $6 \mathrm{~h}$. These values vary, of course, from operator to operator, but this should not strongly influence the qualitative statements presented in this paper. Furthermore, the framework for connection availability analysis using availability maps can be easily implemented taking into account other (specific), repair rates.

The availability maps for six node architectures have been analyzed: the wavelength selective, broadcast and select, and opaque designs for OADMs and OXCs. Figures 14a, 14b, and $14 \mathrm{c}$ show the availability maps for an unprotected connection which traverses OADMs with the WS, B\&S and opaque architectures supporting 40 wavelengths. It is explicit that for both transparent cases the connection availability can achieve 0.999 , but hardly achieves 0.9999 , which means that node or link protection may be needed. It can be seen that 


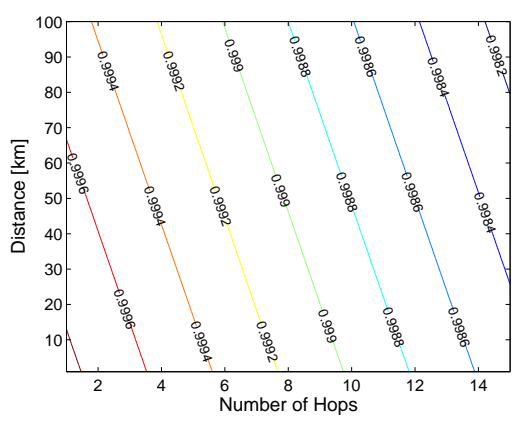

(a) WS

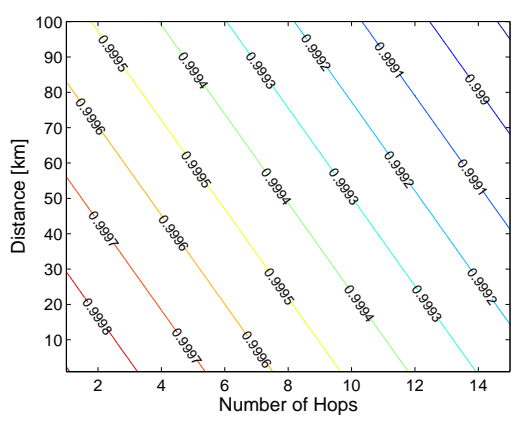

(b) $\mathrm{B} \& \mathrm{~S}$

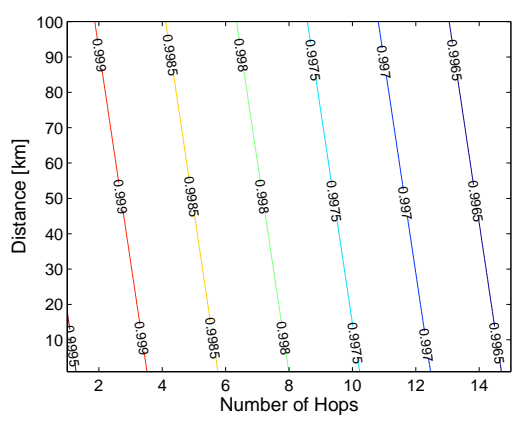

(c) Opaque

Figure 14: Availability maps for an unprotected connection traversing OADM node architectures supporting 40 wavelengths.

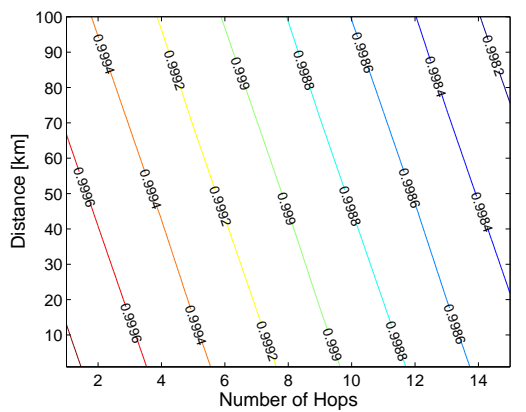

(a) WS

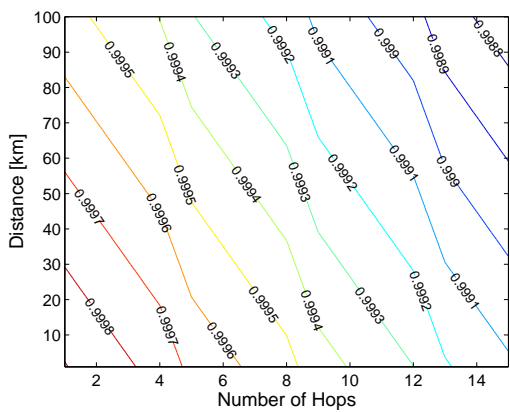

(b) $B \& S$

Figure 15: Availability map for an unprotected connection traversing OADM node architectures supporting 40 wavelengths. The connection is regenerated in $25 \%$ of the nodes.

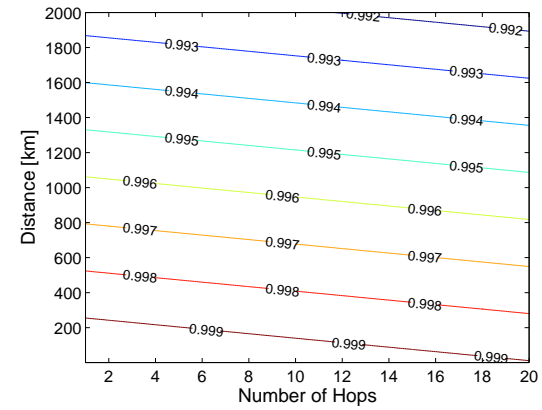

(a) WS

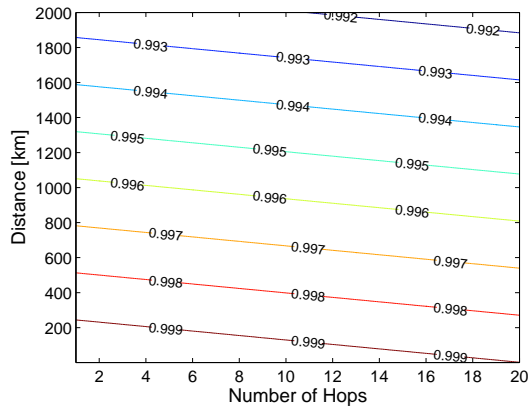

(b) $B \& S$

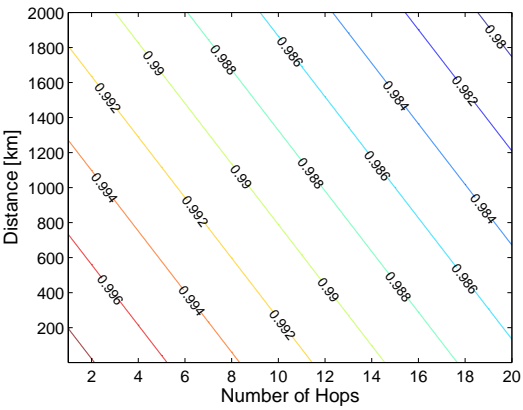

(c) Opaque

Figure 16: Availability map for an unprotected connection traversing OXC node architectures supporting 40 wavelengths and crossconnecting 3 fiber links.

the B\&S OADM performs better than the WS. This is a consequence of the fact that the number of mirrors in the 2D-MEMS switch increases quadratically with the number of supported wavelengths, whereas the failure rate of multiplexers and wavelength blockers increases linearly. This makes the $\mathrm{B} \& \mathrm{~S}$ architecture more attractive in terms of reliability if a high number of wavelengths are supported. If a low number of wavelengths are supported, e.g., 8 wavelengths, the WS architecture performs slightly better than the B\&S. The opaque architecture exhibits the worst performance, such that 0.999 availability cannot be achieved by connections traversing more than 4 hops. In this investigated configuration the opaque architecture would have a performance that is equivalent to the transparent WS architecture if the failure rate of DSW1 could be reduced to about $40 \%$ of its original value. For the connection lengths assumed in this paper (less than $100 \mathrm{~km}$ for connections traversing OADMs), component failures of opaque OADMs dominate over link failures, which is evidenced by the steep curves of the availability map in Figure $14 \mathrm{c}$.

In Figures $15 \mathrm{a}$ and $15 \mathrm{~b}$ the WS and B\&S architectures supporting 40 wavelengths are also considered, but in this case the connection has to be regenerated in $25 \%$ of the nodes. It is noticeable that the impact of regeneration is stronger to the 
B\&S architecture than to the WS. This occurs because the regeneration operation for the WS architecture involves just the transmitter and receiver, whereas for the B\&S architecture it involves two additional amplifiers and couplers.

The availability maps for a connection traversing WS, B\&S and opaque OXCs crossconnecting 3 fiber links and supporting 40 wavelengths can be found in Figures 16a, 16b and 16c. The 80 wavelengths case can be found in Figures 17a, 17b and 17c. It is interesting to note that, in contrast to the OADM case, both transparent architectures perform similarly, even if the number of wavelengths increases. The reason for this is the independence of the WS OXC switch matrix size on the number of supported wavelengths. Since each switch matrix can be separately repaired, increasing the number of wavelengths increases the number of switches inside the optical node, but does not strongly impair the connection availability. The opaque architecture exhibited again an inferior performance compared to the other transparent architectures, and not even 0.999 availability can be achieved. In Figures $18 \mathrm{a}$ and $18 \mathrm{~b}$ the WS and B\&S architectures supporting 40 wavelengths are again considered, but in this case the connection has to be regenerated in $25 \%$ of the nodes. The regeneration operation imposes just a slight degradation to the performance of the $\mathrm{B} \& \mathrm{~S}$ architecture.

Figures 19a, 19b and 19c show the availability maps for a protected connection which traverses transparent WS, B\&S and opaque OXCs crossconnecting 3 fiber links and supporting 40 wavelengths. The connection is regenerated in $25 \%$ of the nodes. The working path is protected by a node-disjoint backup path whose components are exactly the same as for the working path. The smooth steepness of the curves for the transparent cases make clear the dominance of link failures over component failures. It is also worth noting that connections longer than $900 \mathrm{~km}$ could not achieve the 0.99999 availability in any case, which means that there may be a need for a second backup path if carrier-class connection availability requirements are demanded. As for the opaque case, long connections can only achieve 0.9999 availability if they traverse a small number of hops. This points out that for the opaque case path redundancy using idle link capacity may be too costly to compensate for unreliable nodes, and node-internal redundancy may be required.

\section{CONCLUSiON}

This work investigates the joint impact of node architectures and fiber links on the availability of connections in WDM networks. We analyze transparent wavelength selective (WS) and broadcast and select (B\&S) node architectures, of optical add/drop multiplexers (OADMs) and optical crossconnects (OXCs). These transparent solutions are opposed to the opaque case where signals are switched in the electrical domain and thus involve opto-electronic conversion. Depending on the functionality provided at the network node, different components are traversed along the signal path. We distinguish between adding and dropping local traffic, or passing through and signal regeneration of transit traffic. The failure rates of the network components are taken or derived from the literature. Based on the given component failure rate and repair rate, the unavailability of an entire connection can be estimated by summing up the individual unavailability values. Thus the availability can be depicted as a function of the transmission distance and the number of traversed hops. These maps allow to compare the impact of the different node architectures on the connection unavailability. Furthermore, the availability of an actual connection can be easily read off to ensure a desired level of resilience.

The results show that the transparent architectures clearly outperform the investigated opaque scenario. The unavailability of a single digital switch in combination with mandatory receivers and transmitters for optical-electrical-optical (o-e-o) conversion is responsible for the degraded outcome the opaque case. As for an OXC which supports 40 wavelengths and crossconnects 3 fiber links, the considered opaque architecture must have about 10 times lower failure rate to meet the availability region of connections in networks with the investigated transparent nodes. This effect becomes stronger for a higher number of wavelengths. The transparent B\&S OADM architecture allows higher availabilities than the WS case, since the optical switch dominates the unavailability in the WS scenario. For the OXC architecture, the transparent node structures WS and B\&S reveal similar results. The advantage of opaque nodes is to regenerate the optical signal at each hop, which contrasts with transparent architectures in which signal refreshing is not done by default. However, it can be observed that even when providing regeneration at every fourth node in a transparent network, the availability is still similar to the fully transparent case and much higher than in an opaque network. Finally, when adding a backup path as it is done in $1+1$ protection, the availability in both transparent and opaque networks can be significantly improved, but still for some connections a second backup path or node redundancy may be necessary.

\section{ACKNOWLEDGEMENT}

This work has been partially supported by FAPESP, DAAD, and CNPq.

\section{REFERENCES}

[1] Y. Huang, W. Wen, J. Zhang, J. P. Heritage, and B. Mukherjee, "A new link-state availability model for reliable protection in optical WDM networks,' in Proc. ICC'04, Paris, France, June 2004.

[2] Z. Pangi, A. Fumagalli, M. Tacca, and L. Wonsiska, "Impact of OXC failures on network reliability," in Photonics Europe 2004, Strasbourg, France, Apr. 2004.

[3] S. de Maesschalck et al., "Pan-european optical transport networks: an availability-based comparison," Photonic Network Communications, no. 5:3, pp. 203-225, 2003

[4] L. Wosinska, "Reliability study of fault-tolerant multiwavelength nonblocking optical cross connect based on ingaasp/inp laser-amplifier gateswitch arrays," J. Lightwave Technol., vol. 5, no. 10, pp. 1206-1209, Oct. 1993.

[5] L. Wosinska and L. Thylen, "Reliability performance of optical crossconnect switches - requirements and practice,' in $O F C$ '98, San Jose, USA, Feb. 1998.

[6] L. Wosinska, L. Thylen, and R. P. Holmstrom, "Large capacity strictly nonblocking optical cross-connects based on microelectrooptomechanical (MEOMS) switch matrices: Reliability performance anatysis," $J$. Lightwave Technol., vol. 19, no. 8, pp. 1065-1075, Aug. 2001. 


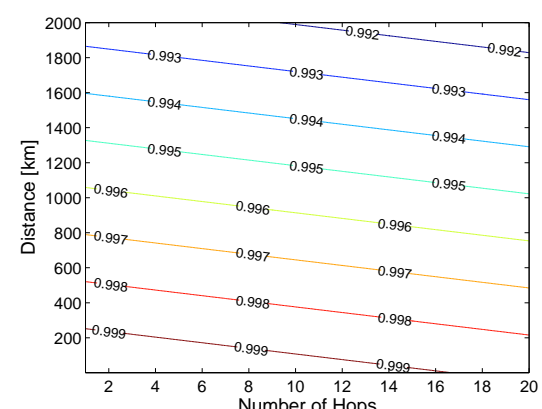

(a) WS

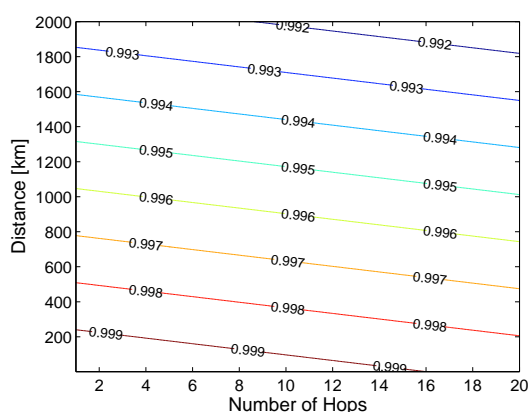

(b) $\mathrm{B} \& \mathrm{~S}$

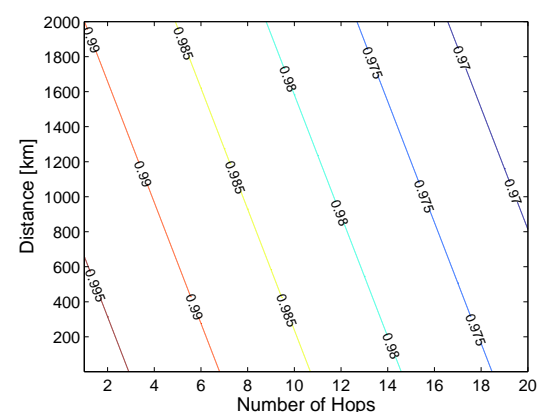

(c) Opaque

Figure 17: Availability map for an unprotected connection traversing OXC node architectures supporting 80 wavelengths and crossconnecting 3 fiber links.

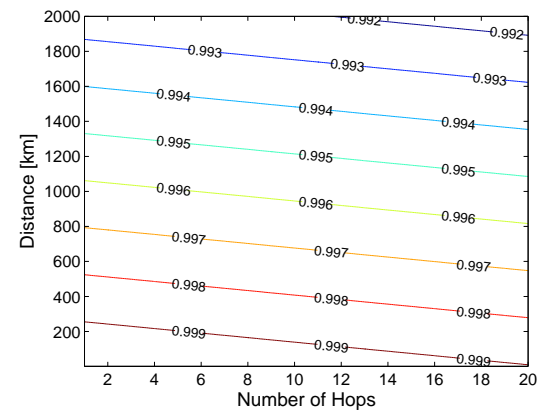

(a) WS

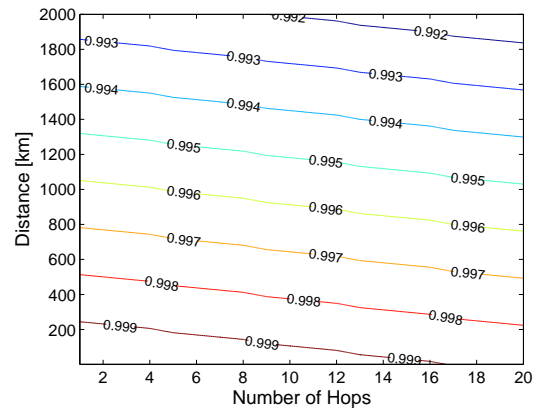

(b) $B \& S$

Figure 18: Availability map for an unprotected connection traversing OXC node architectures supporting 40 wavelengths and crossconnecting 3 fiber links. The connection is regenerated in $25 \%$ of the nodes.

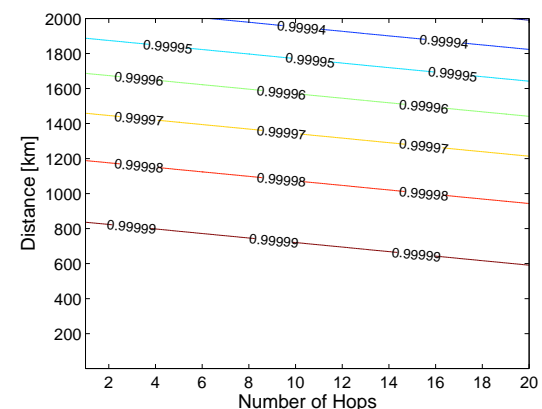

(a) WS

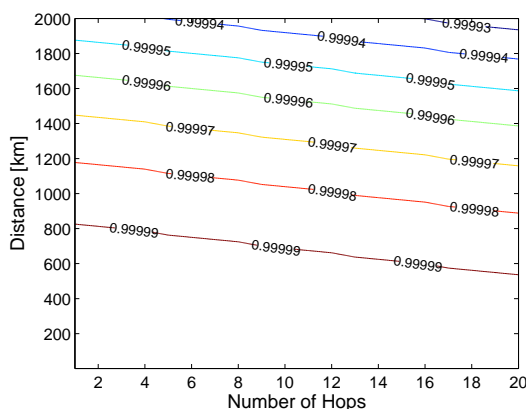

(b) $\mathrm{B} \& \mathrm{~S}$

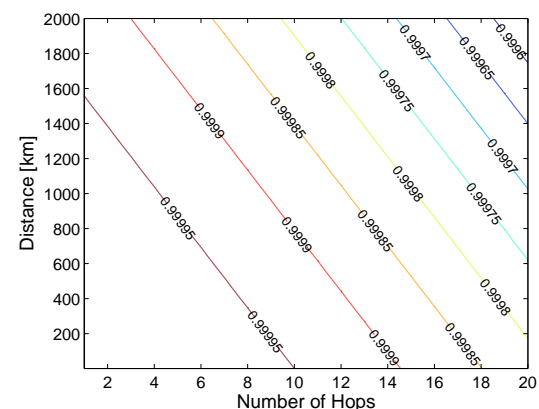

(c) Opaque

Figure 19: Availability map for a $1+1$ protected connection traversing OXC node architectures supporting 40 wavelengths and crossconnecting 3 fiber links. The connection is regenerated in $25 \%$ of the transparent nodes.

[7] M. Lackovic and R. Inkret, "Sensitivity analysis of component failures in the circuit switched WDM network," in ONDM'04, Gent, Belgium, Feb. 2004.

[8] M. Ali, "Shareability in optical networks: Beyond bandwidth optimization," IEEE Commun. Mag., pp. S11-S15, May 2004.

[9] C. Pu, L. Y. Lin, E. L. Goldstein, and R. W. Tkach, "Micro-machined optical add/drop multiplexer with client configurability," in IEEE/LEOS International Conference on Optical MEMS, Kauai, USA, Aug. 2000, pp. 35-36.

[10] P. de Dobbelaere, K. Falta, and S. Gloekner, "Advances in integrated 2D MEMS-based solutions for optical network applications," IEEE Commun. Mag., pp. S16-S23, May 2003.

[11] A. Tzanakaki, I. Zacharapoulos, and I. Tomkos, "Broadband building blocks," IEEE Circuits Devices Mag., pp. 32-37, Mar./Apr. 2004.

[12] T.-W. Yeow, "MEMS optical switches," IEEE Commun. Mag., pp. 158163 , Nov. 2001
[13] M. Vasilev, I. Tomkos, M. Mehenale, J.-K. Rhee, A. Kobyakov, M. Ajgaonkar, S. Tsuda, and M. Sharma, "Transparent ultra-long-haul DWDM networks with "broadcast-and-select" oadm/oxc architecture," J. Lightwave Technol., vol. 21, no. 11, pp. 2661-2697, Nov. 2003.

[14] A. Tzanakaki, I. Zacharopoulos, and I. Tomkos, "Optical add/drop multiplexers and optical cross-connects for wavelength routed networks," in ICTON'03, Warsaw, Poland, June 2003.

[15] M. Jaeger and R. Huelsermann, "Service availability of shared path protection in optical mesh networks," in Proc. ECOC'04, Stockholm, Sweden, 2004.

[16] W. D. Grover, Mesh-Based Survivable Networks. Upper Saddle River, NJ: Prentice Hall, 2004.

[17] M. To and P. Neusy, "Unavailability analysis of long-haul networks," IEEE J. Select. Areas Commun., vol. 12, no. 1, pp. 100-109, Jan. 1994. 